\title{
Situation actuelle de l'irradiation du patient en radiologie dentaire
}

\author{
S. BAECHLER ${ }^{1}$, P. MONNIN ${ }^{1}$, A. AROUA ${ }^{1}$, J.F. VALLEY ${ }^{1}$, M. PERRIER ${ }^{2,3}$, \\ F.R. VERDUN ${ }^{1}$
}

(Manuscrit reçu le 15 janvier 2006, accepté le 11 mai 2006)

RÉSUMÉ L'objectif de cette étude est de faire le point sur l'irradiation du patient dans le domaine de la radiologie dentaire. Les avancées technologiques en imagerie médicale amènent des perspectives intéressantes quant aux possibilités de réduction des doses. Dans ce travail, les doses délivrées aux patients lors d'examens intra-oraux, panoramiques et tomographiques, ont été évaluées sur quelques installations. La dose au patient est généralement estimée en utilisant des indicateurs dosimétriques, tels que le kerma dans l'air à la surface d'entrée du patient (KASE) et le produit kerma surface (PKS). Ces valeurs sont facilement mesurables et permettent d'évaluer la dose efficace pour un patient standard. Le PKS a été mesuré pour un système intra-oral analogique muni de films de sensibilité $D$ et $\mathrm{E} / \mathrm{F}$, ainsi que pour une installation munie d'un détecteur numérique. Afin d'évaluer les doses en radiographie panoramique, le PKS a également été déterminé pour des orthopantomogrammes (OPGs) de différentes générations. Finalement, les doses délivrées aux patients lors d'examens tomographiques en implantologie ont été évaluées à l'aide des mesures du produit kerma longueur (PKL) et de l'indice de dose en tomodensitométrie $\left(\mathrm{CTDI}_{\mathrm{w}}\right)$ pour deux types d'installation : un tomodensitomètre muni des fonctions Dentascan et un système dédié basé sur la technique DVT (Digital Volume Tomography). L'utilisation d'un film E/F au lieu d'un film $D$ permet de réduire le PKS d'un facteur 2 avec une légère augmentation du bruit de l'image. Les systèmes numériques permettent une réduction additionnelle de la dose d'un facteur 6 mais avec une dégradation importante de la résolution spatiale (passage de la FTM à $50 \%$ de $13 \mathrm{~mm}^{-1}$ à $5 \mathrm{~mm}^{-1}$ ). Le PKS mesuré sur les OPGs a démontré que l'ancien système génère une dose trois fois plus élevée qu'un système plus récent. Le système de tomographie dédié permet de réduire la dose au patient d'un facteur 18 en comparaison avec un système CT Dentascan.

ABSTRACT Current situation of doses delivered to the patients in the field of dental radiology.

The purpose of this paper is to present an overview of the doses delivered to the patients in the field of dental radiology. The technology progress in medical imaging will be discussed from a dose perspective. In this work, patient dosimetry has been performed for intra-oral, panoramic and CT dental examinations. Doses were estimated using appropriate dosimetric indicators such as the entrance surface kerma (ESK) and the kerma area product (KAP). These indicators are easily measurable and enable to estimate the effective dose for a standard patient. KAP values were measured for two intra-oral systems using $D$ and $E / F$ speed dental films,

\footnotetext{
Institut Universitaire de Radiophysique Appliquée, Grand-Pré 1, 1007 Lausanne, Suisse.

Policlinique Dentaire de Lausanne, 1011 Lausanne, Suisse.

Adresse actuelle : Cabinet du Dr Michel Perrier, médecin-dentiste, Avenue de Rumine 7, 1005 Lausanne, Suisse.
} 


\begin{abstract}
as well as a digital system based on the CCD technology. In addition, the KAP was measured on three orthopantomograms (OPGs) of various generations. Finally, in order to assess the dose delivered during dental implants planning, the kerma length product (KLP) and the computed tomography dose index $\left(\mathrm{CTDI}_{\mathrm{w}}\right)$ were determined for a CT scanner using the Dentascan protocol and a new DVT (Digital Volume Tomography) dedicated system. Using E/F speed instead of D speed films allowed to reduce the KAP by a factor of 2 without significant loss of image quality. A further dose reduction by a factor of 6 was possible with digital systems but with an important degradation of the spatial resolution (variation of the MTF at $50 \%$ from $13 \mathrm{~mm}^{-1}$ to $5 \mathrm{~mm}^{-1}$ ). KAP measurements on OPGs showed that old systems delivered doses three times higher than a more recent devices. The new dedicated tomographic system enabled a reduction of the patient dose by a factor of 18 when compared with the Dentascan CT system.
\end{abstract}

Keywords : dental radiology / radiation exposure / dose to patient

\title{
1. Introduction
}

La radiologie dentaire est une modalité faiblement irradiante même si les examens dentaires sont très fréquents. Une enquête effectuée en 1998 a démontré que plus de quatre millions d'examens radiologiques dentaires sont pratiqués annuellement en Suisse, soit plus de $40 \%$ du nombre total d'examens à rayons X ; cependant, la contribution relative à la dose collective ne dépasse pas le $1 \%$ (Aroua et al., 2004). Cette technique permet de mettre en évidence un grand nombre de pathologies, d'évaluer la croissance et le développement de la dentition, d'obtenir des informations durant les soins et d'établir rapidement le bilan dentaire d'un patient (EC, 2004). Il existe deux catégories de radiologie dentaire. La plus commune est appelée intra-orale et consiste à prendre le cliché radiologique en plaçant le film à l'intérieur de la cavité buccale. L'autre, appelée extra-orale, regroupe essentiellement la téléradiographie, la radiographie panoramique et la tomographie.

La radiographie intra-orale permet d'évaluer par imagerie de protection les os maxillaire et mandibulaire, et les dents qui leur sont associées. Des radiographies intra-orales de qualité sont obtenues avec des films de sensibilité $\mathrm{E}$ ou F, et permettent d'établir un diagnostic des pathologies dentaires et parodontales. Cette technique reste cependant limitée lorsqu'il s'agit d'évaluer le volume osseux et la localisation précise de repères anatomiques.

La téléradiographie délivre une vue de profil contenant des informations utiles pour l'étude céphalométrique, des typologies faciale et mandibulaire. L'orthopantomogramme (OPG) est la technique de choix dans la pratique journalière du chirurgien oral. Par rapport aux clichés intra-oraux, l'OPG offre une visualisation supérieure et plus globale des structures anatomiques critiques. Cependant, ce cliché bidimensionnel ne donne aucune information sur l'épaisseur 
TABLEAU I

Typologie des examens dentaires intra-oraux. Typology of intra-oral dental exams.

\begin{tabular}{|c|c|c|}
\hline \multicolumn{3}{|c|}{ Radiologie intra-orale } \\
\hline Type d'examen & Zone cible & Indications \\
\hline $\begin{array}{l}\text { Examen périapical } \\
\text { ou rétroalvéolaire } \\
\text { (films périapicaux) }\end{array}$ & $\begin{array}{l}\text { La totalité de la dent (couronne } \\
\text { et racine) ainsi que l'os } \\
\text { alvéolaire associé à la dent. }\end{array}$ & $\begin{array}{l}\text { - Maladies parodontales } \\
\text { - Détection de lésions endodontiques (kystes, abcès, } \\
\text { granulomes...) } \\
\text { - Détection de caries } \\
\text { - Détection de tartre } \\
\text { - Caractérisation d'une lésion traumatique } \\
\text { - Résorption interne et externe } \\
\text { - Sclérose pulpaire } \\
\text { - Imagerie de dents présentes dans la gencive }\end{array}$ \\
\hline $\begin{array}{l}\text { Examen inter-proximal } \\
\text { ou rétrocoronaire } \\
(\text { films «bite-wing ») }\end{array}$ & $\begin{array}{l}\text { La couronne du maxillaire et de } \\
\text { la mandibule sur un même film. }\end{array}$ & $\begin{array}{l}\text { - Détection de caries } \\
\text { - Contrôle du traitement d'une cavité } \\
\text { - Détection de pathologies de la chambre pulpaire }\end{array}$ \\
\hline $\begin{array}{l}\text { Examen occlusal } \\
\text { (films occlusaux) }\end{array}$ & $\begin{array}{l}\text { Les grandes surfaces des } \\
\text { maxillaires (en général } \\
\text { pour préciser une localisation) }\end{array}$ & $\begin{array}{l}\text { - Imagerie de dents présentes dans la gencive } \\
\text { - Localisation de corps étrangers ou de pathologies } \\
\text { - Localisation de lithiases } \\
\text { - Localisation de l'extension du sinus maxillaire } \\
\text { - Localisation de lésions radiovisibles } \\
\text { - Évaluation de traumatismes }\end{array}$ \\
\hline
\end{tabular}

de l'os et limite souvent l'étude de site d'une planification implantaire. Lorsque des risques de mauvais diagnostics et des doutes thérapeutiques subsistent, l'information en trois dimensions est indiquée.

La tomographie conventionnelle est contre-indiquée dans les cas où les lésions ou les sites implantaires sont multiples, ou lorsque des greffes osseuses sont prévues. Le recours à la tomodensitométrie (CT) est alors indiqué. La tomodensitométrie, couplée à un logiciel spécifique de reconstruction d'images, est le premier examen d'imagerie dentaire permettant d'avoir une visualisation directe et isotrope dans les trois dimensions de l'espace. Le CT ne permet pas de mieux caractériser une lésion mais fournit des informations sur son étendue intraet extra-osseuse, notamment une évaluation précise de la morphologie et du volume osseux. Le CT est désormais utilisé pour obtenir des sections maxillaires et mandibulaires en utilisant un logiciel comme le Dentascan qui permet l'obtention d'images des maxillaires sur 3 plans de référence : axial, panoramique et en sections, ainsi que des reconstructions tridimensionnelles. La tomographie conventionnelle est une technique qui peut être aussi précise qu'un CT en se limitant à un site donné et en examinant le patient à des doses d'irradiation beaucoup moins élevées. Les deux techniques sont largement utilisées en chirurgie maxillo-faciale. Les tableaux I et II résument les différents types d'examens de radiologie dentaire ainsi que leurs principales indications. 


\section{TABLEAU II}

Typologie des examens dentaires extra-oraux. Typology of extra-oral dental exams.

\begin{tabular}{|c|c|c|}
\hline \multicolumn{3}{|c|}{ Radiologie extra-orale } \\
\hline Type d'examen & Zone ciblée & Indications \\
\hline $\begin{array}{l}\text { Examen panoramique } \\
(O P G)\end{array}$ & $\begin{array}{l}\text { La totalité des maxillaires sur } \\
\text { un même film }\end{array}$ & $\begin{array}{l}\text { - Caractérisation de dents de sagesse incluses } \\
\text { - Caractérisation de fractures des maxillaires } \\
\text { - Caractérisation de larges lésions au niveau de } \\
\text { la mandibule }\end{array}$ \\
\hline $\begin{array}{l}\text { Examen en projection } \\
\text { ou téléradiographie }\end{array}$ & $\begin{array}{l}\text { Une grande partie du crâne ou } \\
\text { des maxillaires }\end{array}$ & $\begin{array}{l}\text { - Traitements orthodontiques ou chirurgicaux } \\
\text { - Mensurations céphalométriques } \\
\text { - Typologie faciale et mandibulaire } \\
\text { - Évaluation des dents incluses } \\
\text { - Évaluation des maxillaires } \\
\text { - Évaluation de l'articulation temporo- } \\
\text { mandibulaire }\end{array}$ \\
\hline $\begin{array}{l}\text { Examen dento-scan } \\
\text { (tomographie conventionnelle } \\
\text { et tomodensitométrie) }\end{array}$ & $\begin{array}{l}\text { Coupes très fines avec une } \\
\text { résolution longitudinale } \\
\text { excellente }\end{array}$ & $\begin{array}{l}\text { - Chirurgie maxillo-faciale } \\
\text { - Planification d'une pose d'implants } \\
\text { multiples } \\
\text { - Évaluation des sites d'implant } \\
\text { - Localisation des structures anatomiques } \\
\text { - Caractérisation de différentes lésions telles } \\
\text { des suspicions de lésions oncologiques }\end{array}$ \\
\hline
\end{tabular}

Les récentes innovations dans le domaine de la radiologie dentaire sont principalement l'introduction des systèmes numériques en radiographie et le développement de systèmes dédiés en tomographie. Du point de vue de la radioprotection, il s'avère intéressant de comparer les différentes installations disponibles sur le marché et d'évaluer la réduction potentielle des doses en utilisant ces nouveaux systèmes. Ainsi, dans cette étude, la dose délivrée au patient lors d'un examen intra-oral avec un détecteur numérique est comparée à la dose délivrée lors d'un examen avec films. Par ailleurs, des estimations de la dose sont effectuées pour différents systèmes panoramiques, ainsi que pour un tomodensitomètre muni des fonctions Dentascan et pour un système dédié basé sur la technique DVT (Digital Volume Tomography). En radiodiagnostic, l'évaluation de la dose au patient fait généralement appel à des indicateurs dosimétriques, tels que le kerma dans l'air à la surface du patient (KASE) et le produit kerma surface (PKS). Ces grandeurs sont facilement mesurables et permettent d'établir la dose efficace en utilisant des facteurs de conversion appropriés. Finalement, des mesures complémentaires par TLD permettent de déterminer une distribution spatiale de la dose pour une zone du patient bien définie. 


\section{Matériel et méthodes}

\subsection{Les systèmes de détection intra-oraux}

Deux types de films ont été étudiés : le Kodak Ultra Speed DF 58 (classe D) et le Kodak Insight IP 21 (classe E/F). Le kerma à l'entrée du détecteur a été déterminé pour une densité optique nette de 1 afin d'évaluer la sensibilité des films définie comme l'inverse du KASE en mGy, puis pour une densité optique de 1,4 correspondant à un noircissement moyen du film en application clinique. Le contraste moyen a été déterminé en considérant la pente de la courbe sensitométrique entre les densités optiques de 0,5 et 2,5 . Le système numérique utilisé est un RVG Trophy à conversion indirecte. La lumière produite par les rayons $\mathrm{X}$ lors de leur interaction avec le scintillateur est canalisée par des fibres optiques puis transformée en courant d'électrons par des capteurs CCD. Le système RVG Trophy est composé de $1840 \times 1360$ pixels d'une taille de $19,5 \times$ $19,5 \mu \mathrm{m}^{2}$ correspondant à un champ de $36 \times 26,5 \mathrm{~mm}^{2}$. À la sortie du détecteur, les images sont codées sur 12 bits. Une compression logarithmique des données sur 8 bits (256 niveaux de gris) est réalisée directement à la sortie du convertisseur $\mathrm{AD}$. Le détecteur peut être utilisé en mode haute résolution (HR) ou haute sensibilité (HS). Le mode d'acquisition HS est obtenu par la fusion de 4 pixels, augmentant ainsi la sensibilité du système mais dégradant la résolution des images. Pour les deux modes, le kerma à l'entrée du détecteur a été déterminé pour un niveau de gris de 55 qui correspond à un niveau de signal médian dans les conditions habituelles d'exposition.

Les mesures ont été réalisées sur une installation radiologique conventionnelle. La qualité du faisceau a été choisie selon la norme ISO 5799:1991(E) (ISO, 1991). La filtration totale du tube était de $7 \pm 0,5 \mathrm{~mm}$ d'aluminium pur à $99,9 \%$ (filtration interne de $2,5 \mathrm{~mm}$ d'Al + filtration additionnelle de 4,5 mm d'Al) afin de simuler l'atténuation du faisceau par le squelette facial. Une tension de $50 \mathrm{kV}$ a été choisie de sorte que la couche de demi-atténuation (CDA) du faisceau soit de $3,0 \pm 0,2 \mathrm{~mm}$ d'aluminium. Cette tension est inférieure à celles utilisées pour des installations intra-orales $(60-70 \mathrm{kV})$ qui, contrairement aux installations de radiologie conventionnelle, utilisent un redressement mono-alternance et une filtration totale différente réduisant ainsi l'énergie effective pour une tension donnée. Pour les films de classe D et E/F, deux charges de $252 \mathrm{mAs}(400 \mathrm{~mA}$ et $630 \mathrm{~ms}$ ) et $128 \mathrm{mAs}$ ( $320 \mathrm{~mA}$ et $400 \mathrm{~ms}$ ) ont été choisies respectivement. Pour les modes d'acquisition HR et HS du détecteur numérique, deux charges de respectivement $10 \mathrm{mAs}(20 \mathrm{~mA}$ et $500 \mathrm{~ms}$ ) et 2,8 mAs (20 mA et $140 \mathrm{~ms}$ ) ont été utilisées. 
Le KASE a été calculé en multipliant le kerma à l'entrée du détecteur par un facteur 5. Ce facteur a été déterminé en évaluant sur une installation radiologique dentaire à $70 \mathrm{kV}$ (en mono-alternance) le rapport entre le KASE et le kerma à l'entrée du détecteur. Un fantôme de $6 \mathrm{~mm}$ d'aluminium simulant l'atténuation par la mâchoire a été utilisé pour cette mesure, comme indiqué dans la norme DIN 6868-51 (DIN, 1998). Le PKS est obtenu en multipliant le KASE et la surface du champ correspondant à un diamètre de $6 \mathrm{~cm}$. Les facteurs de conversion utilisés pour déterminer la dose efficace, pour une exposition du crâne, à $70 \mathrm{kV}$ et en utilisant une filtration en aluminium de 2,5 mm d'épaisseur, sont de 0,029, 0,022 et $0,027 \mathrm{mSv} \mathrm{Gy}^{-1} \mathrm{~cm}^{-2}$ pour des incidences AP, PA et LAT respectivement (Hart et al., 2002). Un facteur de conversion moyen de $0,03 \mathrm{mSv} \mathrm{Gy}{ }^{-1} \mathrm{~cm}^{-2}$ est utilisé dans cette étude.

\subsection{Les systèmes de détection extra-oraux}

Deux OPG conventionnels, un Philips Ortho-oralix de 20 ans (OPG 1) et un Soredex Cranex de 2 de 8 ans (OPG 2), ainsi qu'un nouvel OPG numérique Planmeca PM2002cc Proline fonctionnant sur la base d'une barrette de CCD (OPG 3) ont fait l'objet de notre étude en radiographie panoramique. Comme indicateur de dose, le PKS est communément utilisé pour les examens OPG. Le facteur de conversion utilisé pour calculer la dose efficace à partir du PKS est de $0,06 \mathrm{mSv} \mathrm{Gy}^{-1} \mathrm{~cm}^{-2}$ (Williams et Montgomery, 2000). En outre, des mesures par TLD ont été effectuées sur l'OPG analogique le plus récent pour évaluer la répartition de la dose à la peau. Ainsi, quatre bandes de 26 pastilles TLD-100 au fluorure de lithium ont été placées sur un fantôme anthropomorphe.

Pour la tomographie dentaire, un CT à 8 barrettes (modèle GE LightSpeed) a été utilisé. Les mesures ont été effectuées en utilisant les paramètres standard pour des examens tomographiques dentaires (protocole Dentascan - $120 \mathrm{kV}, 60$ à $100 \mathrm{mAs}$, pitch de 0,625 , coupes millimétriques). Le système dédié basé sur la technique DVT était un Newtom DVT QR 9000 (voir http://www.qrverona.it). Les mesures ont été réalisées avec une tension de $70 \mathrm{kV}$, un courant de $5 \mathrm{~mA}$ et une durée d'acquisition de $17 \mathrm{~s}$. Cette installation permet d'obtenir non seulement une radiographie de projection similaire à l'image obtenue en OPG, mais aussi des coupes qui sont utilisées lors de la reconstruction de l'image maxillo-faciale. L'acquisition se fait selon la géométrie du «cone beam » et le système enregistre un profil de transmission tous les degrés sur une révolution complète. La dimension du champ dans la direction axiale est de $30 \mathrm{~mm}$ à l'isocentre avec un angle d'ouverture de $14^{\circ}$. La distance foyer-peau du patient est d'environ $430 \mathrm{~mm}$ et la distance entre le foyer et l'axe de rotation est d'environ $680 \mathrm{~mm}$. Les rayons $\mathrm{X}$ sont détectés par un amplificateur de luminance avec une fenêtre d'entrée de 
TABLEAU III

Valeurs caractéristiques des films D (Ultra-speed) et E/F (Insight). Characteristic values of D (Ulta-speed) and E/F (Insight) films.

\begin{tabular}{ccccc}
\hline Film & Développement & $\begin{array}{c}\text { Kerma pour une DO nette } \\
\mathbf{d e} \mathbf{1}[\boldsymbol{\mu} \mathbf{G y}]\end{array}$ & $\begin{array}{c}\text { Sensibilité } \\
{\left[\mathbf{m G y}^{-1}\right]}\end{array}$ & Contraste moyen \\
\hline $\mathrm{D}$ & manuel & 425 & 2,35 & 1,91 \\
& automatique & 400 & 2,50 & 2,05 \\
$\mathrm{E} / \mathrm{F}$ & manuel & 248 & 4,03 & 2,58 \\
& automatique & 170 & 5,87 & 2,05 \\
\hline
\end{tabular}

$20 \times 20 \mathrm{~cm}^{2}$. L'image est enregistrée par un CCD dont la taille de la matrice est de $587 \times 752$ pixels.

Des mesures par TLD ont été réalisées pour les différentes installations tomographiques afin de connaître la distribution de la dose délivrée au patient. Un protocole standard identique à celui utilisé pour l'adulte a été appliqué à un fantôme crânien muni de 2 bandes de TLD. Pour compléter ces mesures, le CTDI a été évalué dans les mêmes conditions que celles utilisées lors des mesures par TLD. Il est alors possible de déterminer le PKL, aussi appelé parfois PDL (produit dose longueur) et, finalement, la dose efficace peut être estimée en utilisant un facteur de conversion de $0,0023 \mathrm{mSv} \mathrm{mGy}{ }^{-1} \mathrm{~cm}^{-1}$ (CEC, 2000).

\section{Résultats}

\subsection{Les systèmes de détection intra-oraux}

Les courbes caractéristiques des deux films Kodak ont été déterminées pour des conditions de développement automatique et manuel. Les sensibilités et les contrastes moyens extraits directement de ces courbes sont donnés dans le tableau III. Les sensibilité des films intra-oraux sont fixées selon la norme ISO 3665:1976 (ISO, 1976) et sont comprises entre 1,37 et $2,71 \mathrm{mGy}^{-1}$ pour la classe D, et entre 2,71 et 10,87 $\mathrm{mGy}^{-1}$ pour la classe E/F. Ainsi, les sensibilités des films Ultra-speed, respectivement Insight, testés dans ce travail correspondent bien à la classe $\mathrm{D}$, respectivement $\mathrm{E} / \mathrm{F}$, indiquées par le fabricant dans les deux conditions de développements. La perte de sensibilité due à un développement manuel est supérieure à $30 \%$ pour le film $\mathrm{E} / \mathrm{F}$ mais négligeable pour le film $\mathrm{D}$. Les deux types de film présentent un contraste moyen identique. Le film E/F est 2,4 fois plus sensible que le film $\mathrm{D}$, mais génère une augmentation significative du bruit de l'image. Un résumé des doses obtenues pour les systèmes intra-oraux est présenté dans le tableau IV en utilisant la méthodologie précisée au paragraphe 2.1. 


\section{TABLEAU IV}

Produit kerma surface et dose efficace pour les systèmes de détection intra-oraux. Kerma-area product and effective dose for intra-oral detection systems.

\begin{tabular}{lcccc}
\hline \multicolumn{1}{|c}{ Système de détection } & $\begin{array}{c}\text { Kerma à l'entrée } \\
\text { du détecteur } \\
{[\boldsymbol{\mu} \mathbf{G y}]}\end{array}$ & $\begin{array}{c}\text { Kerma d'entrée } \\
\text { à la peau } \\
{[\mathbf{m G y}]}\end{array}$ & $\begin{array}{c}\text { Produit kerma } \\
\text { surface } \\
{\left[\mathbf{m G y ~ c m}^{2}\right]}\end{array}$ & $\mathbf{E}$ \\
\hline Film Kodak Ultra-speed (D) & 450 & 2,25 & 64 & 1,9 \\
\hline Film Kodak Insight (E/F) & 190 & 0,95 & 27 & 0,81 \\
\hline RVG Trophy en mode HR & 29 & 0,15 & 4,2 & 0,13 \\
\hline RVG Trophy en mode HS & 6 & 0,03 & 0,85 & 0,03 \\
\hline
\end{tabular}

TABLEAU V

Évaluation du Produit kerma surface et de la dose efficace pour les trois OPG. Kerma-area product and effective dose for three OPGs.

\begin{tabular}{lccccccc}
\hline \multicolumn{1}{|c}{ Installation } & $\begin{array}{c}\text { Tension } \\
{[\mathbf{k V}]}\end{array}$ & $\begin{array}{c}\text { Courant } \\
{[\mathbf{m A}]}\end{array}$ & $\begin{array}{c}\text { Temps } \\
{[\mathbf{s}]}\end{array}$ & $\begin{array}{c}\text { PKL } \\
{[\mathbf{m G y ~ m m}]}\end{array}$ & $\begin{array}{c}\text { Long/Large } \\
\text { Fente }[\mathbf{m m}]\end{array}$ & $\begin{array}{c}\text { PKS } \\
{\left[\mathbf{G y ~ c m}^{2}\right]}\end{array}$ & $\begin{array}{c}\mathbf{E} \\
{[\mu \mathbf{S v}]}\end{array}$ \\
\hline OPG 1 (20 ans) $^{\text {a) }}$ & 75 & 17.3 & 20 & 130,0 & $145,0 / 5,0$ & 0,19 & 11 \\
OPG 2 (8 ans) $^{\text {a) }}$ & 71 & 6 & 20 & 55,0 & $125,0 / 5,4$ & 0,069 & 4,1 \\
OPG 3 (récent) $^{\text {a) }}$ & 68 & 7 & 18 & 73,0 & $131,5 / 5,3$ & 0,096 & 5,8 \\
Référence $^{\text {b) }}$ & & & & 65 & & 0,11 & \\
\hline
\end{tabular}

Nombre d'années depuis la première mise en service.

b) Valeur moyenne donnée dans la littérature (Williams et Montgomery, 2000).

\subsection{Systèmes de détection extra-oraux}

\subsubsection{Orthopantomogrammes}

Les résultats des mesures pour les trois OPGs sont résumés dans le tableau V. Les PKS obtenus pour les installations les plus récentes sont 2 fois inférieurs à la valeur obtenue pour l'ancienne installation. En effet, pour produire une qualité d'image suffisante au niveau clinique, l'ancien système nécessite l'utilisation d'une tension et d'un courant plus élevés. De plus, la longueur de la fente est supérieure. La distribution des doses obtenues pour l'OPG 2 par mesures TLD sur le fantôme anthropomorphe montre une dose moyenne de $0,3 \mathrm{mGy}$ à la mâchoire (pic à $0,8 \mathrm{mGy})$, de $0,2 \mathrm{mGy}$ à la thyroïde et de $0,1 \mathrm{mGy}$ au cristallin.

\subsubsection{Installations tomographiques}

Les résultats relatifs à la distribution des doses sont donnés dans le tableau VI. Concernant la mesure du CTDI $\mathrm{w}_{\mathrm{w}}$, le Newton DVT donne 4,2 mGy en utilisant le fantôme de $16 \mathrm{~cm}$ de diamètre. Pour une longueur d'irradiation de $3 \mathrm{~cm}$ à 


\section{TABLEAU VI}

Distribution des doses et évaluation de la dose efficace pour le Newtom DVT et le CT Dentascan. Dose distribution and effective dose for the Newtom DVT and the CT Dentascan.

\begin{tabular}{|c|c|c|c|c|c|c|}
\hline \multirow[t]{2}{*}{ Installation } & \multicolumn{3}{|c|}{ Dose absorbée [mGy] } & \multirow{2}{*}{$\begin{array}{c}\mathrm{CTDI}_{\mathrm{W}} \\
{[\mathrm{mGy}]}\end{array}$} & \multirow{2}{*}{$\begin{array}{c}\text { PKL } \\
{[\mathrm{mGy} \mathrm{cm]}}\end{array}$} & \multirow{2}{*}{$\begin{array}{c}\mathbf{E} \\
{[\mu \mathrm{Sv}]}\end{array}$} \\
\hline & Thyroïde & Mâchoire & Cristallin & & & \\
\hline Newtom DVT a) & 0,6 & 4,0 & 2,1 & 4,2 & 12.6 & 29 \\
\hline CT Dentascan b) & 3,5 & 32 & 2,5 & $19 / 24$ & $106 / 118$ & $250 / 270$ \\
\hline
\end{tabular}

${ }^{a)}$ Examen combiné mandibule/maxillaire.

b) Examens distincts mandibule/maxillaire.

l'isocentre, cet examen donne un PKL de $12.6 \mathrm{mGy} \mathrm{cm}$. Ceci conduit à une dose efficace de $29 \mu \mathrm{Sv}$. Pour le Dentascan, le $\mathrm{CTDI}_{\mathrm{w}}$ est de $19 \mathrm{mGy}$ pour un examen de la mandibule, le PKL de $106 \mathrm{mGy} \mathrm{cm}$ et la dose efficace de $250 \mu \mathrm{Sv}$. Une mesure similaire a été réalisée pour le maxillaire supérieur et donne un $\mathrm{CTDI}_{\mathrm{w}}$ de $24 \mathrm{mGy}$, un PKL de $118 \mathrm{mGy} \mathrm{cm}$ et une dose efficace de $270 \mu \mathrm{Sv}$.

\section{Discussion}

Concernant la technique intra-orale, le PKS obtenu est de $0,024 \mathrm{~Gy} \mathrm{~cm}^{2}$ lors de l'utilisation d'un film E/F, et de $0,056 \mathrm{~Gy} \mathrm{~cm}^{2}$ pour un film D. Ainsi, le film E/F offre la possibilité de réduire la dose d'un facteur 2. Dans une étude du NRPB portant sur 471 installations avec des films E/F et une tension comprise entre 45 et $55 \mathrm{kV}$, le PKS moyen obtenu est de $0,11 \mathrm{~Gy} \mathrm{~cm}^{2}$ avec des valeurs comprises entre 0,018 et $0,57 \mathrm{~Gy} \mathrm{~cm}^{2}$ (Napier, 1999). Le PKS résultant de notre étude en appliquant les mêmes conditions se situe à l'intérieur de cet intervalle. Pour 839 autres installations de l'échantillon du NRPB, la tension appliquée se situait entre 60 et $70 \mathrm{kV}$; le PKS moyen est alors de $0,05 \mathrm{~Gy} \mathrm{~cm}^{2}$. En utilisant le facteur de conversion de $0,03 \mathrm{mSv} \mathrm{Gy}{ }^{-1} \mathrm{~cm}^{-2}$ propre à la radiologie intra-orale, un cliché obtenu à l'aide d'un système analogique, avec un film $\mathrm{E} / \mathrm{F}$ et une tension de 60 à $70 \mathrm{kV}$, délivre une dose efficace de l'ordre 1,5 $\mu \mathrm{Sv}$. L'utilisation de systèmes numériques tels que le Trophy permet de réduire le niveau d'exposition d'un facteur 6 en mode HR et 30 en mode HS par rapport à l'utilisation d'un film $\mathrm{E} / \mathrm{F}$.

En radiologie dentaire panoramique, le PKS moyen obtenu dans ce travail est d'environ $0,12 \mathrm{~Gy} \mathrm{~cm}^{2}$. Cette valeur est comparable au PKS moyen de $0,11 \mathrm{~Gy} \mathrm{~cm}^{2}$ mesuré à l'aide de TLD par Williams et Montgomery sur 16 installations panoramiques (Williams et Montgomery, 2000). Une étude récente de Tierris et al. réalisée sur 62 installations rapporte un PKS moyen similaire de 
$0,10 \mathrm{~Gy} \mathrm{~cm}^{2}$ et une valeur correspondant au $75 \%$ de la répartition de $0,12 \mathrm{~Gy} \mathrm{~cm}^{2}$ pour l'examen standard d'un adulte de sexe masculin (Tierris et al., 2004). L'utilisation du système numérique Planmeca ne s'est pas traduite par une diminution de la dose par rapport au système Soredex. Au niveau de la comparaison du risque radiologique, la situation est plus complexe puisque l'irradiation de la thyroïde varie sensiblement d'une installation ou d'un positionnement à l'autre. Pour tenir compte des différentes situations d'irradiation, un facteur de conversion moyen de $0,06 \mathrm{mSv} \mathrm{Gy}^{-1} \mathrm{~cm}^{-2}$ est proposé dans la littérature (Williams et Montgomery, 2000). Un examen sur un OPG récent délivre ainsi une dose efficace d'environ $5 \mu \mathrm{Sv}$ au patient. Une certaine prudence s'impose lors de la comparaison des doses efficaces en radiologie dentaire avec celles d'autres examens radiologiques. En effet, les facteurs de pondérations $\mathrm{w}_{\mathrm{T}}$ sont très faibles dans la région crânienne, et la dose aux glandes salivaires est souvent omise dans le calcul. Ainsi, en considérant la dose aux glandes salivaires, Ludlow et al. ont constaté que la dose efficace pour un examen panoramique passait de $6,2 \mu \mathrm{Sv}$ à $22 \mu \mathrm{Sv}$ (Ludlow et al., 2003).

Au niveau des acquisitions tomographiques, les mesures par TLD réalisées dans cette étude démontrent que le système Newtom DVT permet de réduire significativement les doses à la glande thyroïde et à la mâchoire en comparaison avec le CT Dentascan. Dans le cas du Newtom DVT, Cohen et al., Ludlow et al., ainsi que Mah et al., obtiennent des valeurs similaires pour la thyroïde, mais deux à trois fois inférieures au niveau des mâchoires (Cohen et al., 2002 ; Ludlow et al., 2003; Mah et al., 2003). Ces écarts sont principalement dus à l'incertitude associée au positionnement du fantôme anthropomorphe et au choix du protocole d'acquisition des images. Les mesures effectuées sur l'installation Newtom DVT fournissent un $\mathrm{CTDI}_{\mathrm{w}}$ de 4,2 $\mathrm{mGy}$ et un PKL d'environ 12,6 $\mathrm{mGy} \mathrm{cm}$ dans des conditions standard pour un examen combiné de la mandibule et du maxillaire. L'utilisation du facteur de conversion en CT pour un examen de la tête $\left(0,0023 \mathrm{mSv} \mathrm{mGy}^{-1} \mathrm{~cm}^{-1}\right)$ permet d'estimer la dose efficace à $29 \mu \mathrm{Sv}$. À l'aide de TLD placés sur différents sites anatomiques d'un fantôme anthropomorphe, Ludlow et al. ainsi que Mah et al. ont obtenu une dose efficace comparable, respectivement de $40 \mu \mathrm{Sv}$ et $50 \mu \mathrm{Sv}$ (Ludlow et al., 2003; Mah et al., 2003). En considérant la dose délivrées aux glandes salivaires, la dose efficace se situe entre 80 et $110 \mu \mathrm{Sv}$ (Cohen et al., 2002; Ludlow et al., 2003). Le CT Dentascan délivre des doses nettement plus élevées puisque la somme des doses obtenues pour un examen de la mandibule et du maxillaire est de l'ordre de $520 \mu \mathrm{Sv}$. D'après Mah et al., les doses efficaces pour un examen maxillo-facial en $\mathrm{CT}$ se situent entre 125 et $530 \mu \mathrm{Sv}$ pour la mandibule, et entre 20 et $660 \mu \mathrm{Sv}$ pour le maxillaire, sans prendre en compte la dose aux glandes salivaires (Mah et al., 2003). 
TABLEAU VII

Dose efficace par examen pour divers examens radiologiques dentaires et autres. Effective dose per exam for various dental and other radiological exams.

\begin{tabular}{cccc}
\hline & Examen & Système & $\begin{array}{c}\text { Dose efficace par examen } \\
{[\mu \text { Sv }]}\end{array}$ \\
\hline Dentaires & Intra-oral & Film & 1 \\
& & Détecteur numérique CCD & 0,15 \\
& Panoramique & OPG & 5 \\
& Tomographique & CT dédié (DVT) & 29 \\
\hline Autres & CT (Dentascan) & 520 \\
& Thorax & Film & 20 \\
& Abdomen & Film & 1500 \\
\hline
\end{tabular}

\section{Conclusion}

L'indicateur de dose que l'on peut utiliser pour la radiologie intra-orale ou l'OPG est le produit kerma surface (PKS). Lors de l'utilisation d'un film E/F, le PKS moyen obtenu est d'environ $0,05 \mathrm{~Gy} \mathrm{~cm}{ }^{2}$. L'utilisation de systèmes numériques tels que Trophy permet de diminuer le niveau d'exposition d'un facteur 6 à 30 suivant le mode d'acquisition choisi. En radiologie dentaire panoramique, le PKS est d'environ $0,1 \mathrm{~Gy} \mathrm{~cm}^{2}$. Ainsi, en terme d'exposition, une acquisition OPG correspond approximativement à 2 clichés intra-oraux. En ce qui concerne le risque radiologique, un cliché intra-oral avec un film de E/F délivre une dose efficace d'environ 1 à $1,5 \mu \mathrm{Sv}$ tandis qu'un OPG moderne délivre environ $5 \mu \mathrm{Sv}$. En tomographie dentaire, il apparait que l'utilisation du $\mathrm{CTDI}_{\mathrm{w}}$ permet une comparaison des modalités des différents systèmes. L'utilisation de l'installation Newtom DVT pour un examen combiné des deux mâchoires permet de réduire la dose efficace d'un facteur 18 par rapport à un examen CT Dentascan de la mandibule et du maxillaire. En effet, pour ce système basé sur la technique du « cone beam », la dose efficace est de $29 \mu \mathrm{Sv}$ alors qu'elle s'élève à $520 \mu \mathrm{Sv}$ pour un CT dentaire.

Le tableau VII présente un résumé des risques radiologiques rencontrés en radiologie dentaire ainsi que pour d'autres examens fréquents en radiodiagnostic médical. Comme la dose aux glandes salivaires n'est pas prise en compte dans ce travail, la comparaison des doses efficaces en dentaire avec celles d'autres examens nécessite une certaine prudence. Un examen dentaire panoramique représente l'équivalent de trois à quatre clichés intra-oraux; une radiographie du thorax en représente environ 15 . Un examen tomographique avec un système dédié tel que le Newtom DVT équivaut à environ six clichés panoramiques, alors qu'un 
CT Dentascan en représente plus de 100. À l'exception des examens tomographiques, les risques en radiologie dentaire sont nettement inférieurs à ceux auxquels le patient est exposé lors d'une radiographie au niveau du tronc.

\section{RÉFÉRENCES}

Aroua A., Buchillier-Decka I., Dula K., Nedjadi Y., Perrier M., Vader J.-P., Valley J.-F. (2004) Radiation Exposure in Dental Radiology: A 1998 Nation-wide survey in Switzerland, Dentomaxillofac Radiol. 33, 211-219.

Cohen M., Kemper J., Möbes O., Pawelzik J., Mödder U. (2002) Radiation dose in dental radiology, Eur. Radiol. 12, 634-637.

CEC (2000) Commission of the European Communities, EUR 16262 EN, European guidelines on quality criteria for computed tomography, Luxembourg .

DIN (1998) Deutsches Institut für Normung, DIN 6868-51, Image quality assurance in X-ray diagnostics - Part 51: Acceptance testing of dental radiographic equipment; rules for the inspection of image quality after installation, maintenance and modification, Berlin.

EC (2004) Office for Official Publications of the European Communities, RP-136, European guidelines on radiation protection in dental radiology, Luxembourg.

Hart D., Jones D.G., Wall B.F. (2002) Estimation of effective dose in diagnostic radiology from entrance surface dose and dose area product measurements, Document NRPB-R262, Chilton UK.

ISO (1976) International Organization for Standardization, ISO 3665-1976, Photography - Intra-oral dental radiographic film - Specification, Geneva, ISO.

ISO (1991) International Organization for Standardization, ISO 5799-1991(E), Photography - Directexposing medical and dental radiographic film/process systems - Determination of ISO speed and ISO average gradient, Geneva, ISO.

Ludlow J.B., Davies-Ludlow L.E., Brooks S.L. (2003) Dosimetry of two extraoral direct digital imaging devices: NewTom cone beam CT and Orthophos Plus DS panoramic unit, Dentomaxillofac Radiol. 32, 229-234.

Mah J.K., Danforth R.A., Bumann A., Hatcher D. (2003) Radiation absorbed in maxillofacial with a new dental computed tomography device, Oral. Surg. Oral. Med. Oral. Pathol. Oral. Radiol. Endod. 96, 508-513.

Napier I.D. (1999) Reference doses for dental radiography, Br. Dent. J. 186, 392-396.

Tierris C.E., Yakoumakis E.N., Bramis G.N., Georgiou E. (2004) Dose area product reference levels in dental panoramic radiology, Radiat. Prot. Dosim. 111, 283-287.

Williams J.R., Montgomery A. (2000) Measurement of dose in panoramic dental radiology, Br. J. Radiol. 73, 1002-1006. 